\title{
Erratum to: From Keynes to Piketty
}

\section{Peter de Haan}

\section{Erratum to:}

Bibliography from letter S-Z which was missing has been updated in the printed version.

DOI 10.1057/978-1-137-60002-8. 